\title{
Universiteit
}

Leiden

The Netherlands

\section{Cortisol-induced impairments of working memory require acute sympathetic activation.}

Elzinga, B.M.; Roelofs, K.

\section{Citation}

Elzinga, B. M., \& Roelofs, K. (2005). Cortisol-induced impairments of working memory require acute sympathetic activation. Behavioral Neuroscience, 119, 98-103. Retrieved from https://hdl.handle.net/1887/14220

Version: $\quad$ Not Applicable (or Unknown)

License: $\quad$ Leiden University Non-exclusive license

Downloaded from: https://hdl.handle.net/1887/14220

Note: To cite this publication please use the final published version (if applicable). 


\title{
Cortisol-Induced Impairments of Working Memory Require Acute Sympathetic Activation
}

\author{
Bernet M. Elzinga and Karin Roelofs \\ University of Leiden
}

\begin{abstract}
The present study assessed whether the effects of cortisol on working memory depend on the level of adrenergic activity (as measured by sympathetic activation) during memory performance. After exposure to a psychosocial stress task, participants were divided into cortisol responders and nonresponders. Cortisol responders showed working memory impairments during the psychosocial stress phase, when cortisol and adrenergic activity were enhanced, whereas nonresponders did not. During recovery, however, when cortisol levels were elevated but adrenergic activity was normalized, working memory of responders did not differ from that of nonresponders. Among several stress measures, cortisol was the only significant predictor for working memory performance during stress. These findings suggest that adrenergic activation is essential for the impairing effects of stress-induced cortisol on working memory.
\end{abstract}

It is well established that glucocorticoid (GC) hormones released from the adrenal cortex during stressful or emotionally arousing experiences play an important role in the regulation of cognitive functions (see De Kloet, Oitzl, \& Joëls, 1999; Lupien \& Lepage, 2001; Roozendaal, 2002). Several studies in humans have documented a deleterious effect of GCs on the retrieval of previously learned material, although they may enhance long-term consolidation of (emotional) material (Buchanan \& Lovallo, 2001; Cahill, Gorski, \& Le, 2003; Kirschbaum, Wolf, May, Wippich, \& Hellhammer, 1996; Newcomer, Craft, Hershey, Askins, \& Bardgett, 1994; Newcomer et al., 1999; De Quervain, Roozendaal, Nitsch, McGaugh, \& Hock, 2000). Working memory, the cognitive mechanism that allows us to keep a limited amount of information active for a limited period of time (Baddeley, 1996), is one of the memory functions that is particularly sensitive to GCs. Three studies in humans have consistently shown cortisol administration to impair working memory, without having effects on declarative memory and sustained attention (Lupien, Gillin, \& Hauger, 1999; Wolf et al., 2001; Young, Sahakian, Robbins, \& Cowen, 1999), suggesting that cortisol specifically impairs the working memory component.

Evidence from lesion, pharmacological, and preclinical studies indicates that the prefrontal cortex (PFC) is a key brain structure in working memory (Arnsten, 1998; Fuster, 1997). This is supported by neuroimaging studies showing a significant linear relationship between the increase in working memory load and the degree of

Bernet M. Elzinga and Karin Roelofs, Section of Clinical and Health Psychology, University of Leiden, Leiden, the Netherlands.

This study was supported by Veni Grant 451-02-116 from the Netherlands Organization for Scientific Research awarded to Bernet M. Elzinga. We thank B. Poelmans, J. Meulman, R. Siekman, and F. Vulker for their assistance during data collection, and R. de Kloet, M. Oitzl, and P. Spinhoven for their valuable theoretical comments.

Correspondence concerning this article should be addressed to Bernet M. Elzinga, University of Leiden, Section of Clinical and Health Psychology, P. O. Box 9555, Leiden 2300 RB, the Netherlands. E-mail: elzinga@ fsw.leidenuniv.nl activation observed in the dorsolateral PFC (Braver et al., 1997, Jansma, Ramsey, Coppola, \& Kahn, 2000; Rypma, Prabhakaran, Desmond, Glover, \& Gabrieli, 1999; Veltman, Rombouts, \& Dolan, 2003). The PFC is a significant target for the negativefeedback actions of circulating GCs (Sanchez, Young, Plotsky, \& Insel, 2000). Moreover, animal research has shown that chronic corticosterone administration and chronic behavioral stress can lead to dendritic reorganization in the medial PFC (Radley et al., 2004; Wellman, 1993), and that exposure to stress-induced cortisol elevation can impair prefrontal behavioral control (Lyons, Lopez, Yang, \& Schatzberg, 2000).

Recent findings from studies in animals suggest that the influence of GCs on memory functioning depends on the level of training-induced emotional arousal (Okuda, Roozendaal, \& McGaugh, 2004) and/or adrenergic activity (Roozendaal, 2000) during memory performance. Okuda and colleagues (2004) showed that only rats that were not habituated to an experimental context (i.e., increased novelty-induced emotional arousal) showed an inverted-U-shaped relationship between dose of corticosterone and object recognition, whereas corticosterone did not affect object recognition of rats that were habituated to the experimental context (i.e., decreased novelty-induced emotional arousal). The activation of noradrenergic mechanisms in the basolateral complex of the amygdala (BLA) may be a critical component in mediating the effects of emotional arousal on the effects of GCs on memory functions in rats, by interacting with several other brain regions, including the hippocampus and the PFC (McGaugh \& Roozendaal, 2002; Roozendaal, 2000). Consistent with the suggested role of the BLA in mediating the effects of GCs on prefrontal cognitive functions, GC treatments induced impairments in the performance of a spatial memory task, a task that depends on the medial PFC, whereas BLA lesions blocked the impairments induced by GC administration (Roozendaal, McReynolds, \& McGaugh, 2004). Likewise, systemic injections of the centrally acting $\beta$-adrenoceptor antagonist propranolol prevented corticosteroneinduced memory impairment (Roozendaal et al., 2004), suggesting that the modulation of working memory by cortisol elevation requires noradrenergic activation. To our knowledge, the role of 
noradrenergic activation in mediating the effects of GCs on working memory has not been investigated in humans.

To investigate whether the effects of stress-induced cortisol elevations on working memory depend on the level of adrenergic activity (as measured by sympathetic autonomic activation) during working memory performance, we randomly assigned participants to either a psychosocial stress (Trier Social Stress Task [TSST]) or a reading condition, after which working memory (Digit Span forward and backward; Wechsler, 1987) was assessed at baseline (i.e., when both cortisol levels and adrenergic activity are low), during acute psychosocial stress (when both cortisol levels and adrenergic activity are high), and after psychosocial stress (i.e., when cortisol levels are still elevated, but adrenergic activity is low). We hypothesized that working memory would specifically be impaired under conditions of both elevated cortisol levels and increased adrenergic activation.

\section{Method}

\section{Participants}

Forty-four college students, who participated for financial reward or course credit, were randomly assigned to either the stress condition $(n=$ $22 ; 12$ men and 10 women; mean age $=21.33$ years, $S D=4.21$ ) or control reading condition $(n=22 ; 11$ men and 11 women; mean age $=21.10$ years, $S D=1.84$ ). Participants were recruited from the University of Leiden through announcements. Exclusion criteria were as follows: any Axis I psychiatric disorder, including substance abuse (DSM-IV); any clinically significant medical disease; use of medication (including oral contraceptives); and being less than 18 or more than 37 years of age. All women were tested in the late luteal phase (Days 21-25) of their menstrual cycle according to self-report. The luteal phase was chosen because there are indications that during this phase, stress-induced cortisol levels do not differ between men and women (see Kirschbaum, Kudielka, Gaab, Schommer, \& Hellhammer, 1999). Participants were asked to minimize physical exercise during the hour preceding the experiment and not to take large meals, coffee, drinks with low $\mathrm{pH}$, or cigarettes, because these variables can have an influence on cortisol levels. All participants signed informed consent.

\section{Tasks}

TSST. This psychosocial challenge test, which mainly consists of a free speech and mental arithmetic task of 15 min duration, has repeatedly been found to induce significant endocrine and cardiovascular responses (Kirschbaum, Pirke, \& Hellhammer, 1993). Participants received instructions in which they were told that they would be taking on the role of a job applicant for the position of research assistant at the University of Leiden. Participants were given $5 \mathrm{~min}$ to prepare a 5-min free speech to an audience of three psychologists, one of whom acted as chairman. They were told that the speech would be videotaped, that the psychologists were trained to monitor nonverbal behavior, that a voice-frequency analysis of nonverbal behavior would be performed, and that the speech would be critiqued on content and presentation style. Following preparation time, the audience entered the room and obviously switched on the camera and microphone. Participants were instructed to stand in front of a table with the audience sitting at the other side, and the chairman asked the participant to describe his or her qualifications for the job. Participants were expected to use the entire $5 \mathrm{~min}$ for the speech, as described by Kirschbaum et al. (1993). For the mental arithmetic task, participants were instructed to serially subtract 13, starting with 1587 (i.e., 1587 - 13, 1574 - 13, etc.). The audience responded to any mistakes by instructing participants to start again from the beginning.
Working memory task. Working memory was measured with the Digit Span subtest of the Wechsler Adult Intelligence Scale-Revised (Wechsler, 1987). Five series of numbers of increasing length (from 4 to 8 in the forward condition, and 3 to 7 in the backward condition) were read to each participant at the rate of one digit per second. Participants had to repeat the numbers in the same order (forward condition) or in reversed order (backward condition). Each set length was tested twice. For each correctly repeated digit set, the number of digits was added up and the scores for the forward and backward conditions were combined, so that scores could range from 0 to 120 . Three parallel versions of the working memory task were administered (see Figure 1): one at baseline (no audience present), one immediately after the stress task in front of the audience, and one after the stress task (no audience present). Administration of the three versions was counterbalanced.

\section{Physiological Assessments}

All physiological assessments were obtained over a 95-min period at eight assessment points: $-45,-30,-15,-5,+10,+20,+35$, and +50 min with reference to the start of the stressor.

Cortisol. Cortisol samples were obtained with Salivette collection devices (Sarstedt, Rommelsdorf, Germany). Saliva samples were stored at $-20{ }^{\circ} \mathrm{C}$ before assay. Biochemical analysis of free cortisol in saliva was performed with a competitive electrochemiluminescence immunoassay (Elecsys 2010; Roche Diagnostics, Laval, Quebec, Canada), as described elsewhere (van Aken, Romijn, Miltenburg, \& Lentjes, 2003).

Heart rate. Heart rate was recorded continuously by an ambulatory monitoring system (Version 3.6; Vrije Universiteit Amsterdam), a small battery-powered device for ambulatory recording. It was measured with three $\mathrm{Ag}-\mathrm{AgCl}$ disposable electrodes (ConMed, Utica, NY) placed just above the sternum, at the left side of the chest and at the bottom right side of the chest. For each participant, heart rate was averaged for $2 \mathrm{~min}$, starting from a marker given at each of the eight assessment points.

Blood pressure. Systolic and diastolic blood pressure were measured from the nondominant arm with a blood pressure monitor (Model 705CP; Omron, Bannockburn, IL).

Subjective measures. Subjective measures of anxiety, nervousness, and feelings of insecurity were assessed on a visual analogue scale ranging from 0 to 10 .

Procedure. Participants arrived at the laboratory at 9 a.m., after which the ambulatory monitoring system device was connected and checked by the experimenter. Participants were randomly assigned to the stress or reading condition. At -45 min with reference to the start of the stressor, a first series of physiological and subjective measures were assessed. At -35 min, the working memory task was administered for the first time (baseline), followed by a few additional tasks, results of which will be reported elsewhere (Roelofs, Elzinga, \& Rotteveel, 2004), and three series of physiological and subjective measures at $-30,-15$, and $-5 \mathrm{~min}$. Subsequently, the TSST took place between 0 and +15 min. Immediately after the TSST, the chairman administered the second working memory task while the participant was standing in front of the audience, so that the social stress context remained present during the second administration of the working memory task (stress period). At +35 , min the audience left the room. At +50 min, working memory performance was assessed for a third time (recovery period), followed by the last physiological and subjective measures. After finishing the experiment, the audience returned for a short debriefing

\section{Statistical Analysis}

To compare the experimental condition and the control condition on working memory performance and on the physiological and subjective stress responses, we used analyses of variance (ANOVAs) for repeated measures, with condition (stress vs. control) as a between-subjects factor 
and time as a within-subjects factor. Analyses were performed with SPSS 11.5 (SPSS, Chicago, IL). The criterion for statistical significance was $p<$ .05 , two-tailed.

\section{Results}

\section{Stress Induction}

A separate ANOVA with repeated measures for the physiological and subjective stress measures showed significant increases on all stress measures over time in the stress, but not in the control, condition: significant interactions for Group $\times$ Time for heart rate, $F(7,287)=6.38, p<.0001$; systolic blood pressure, $F(7,294)=$ 5.31, $p<.0001$; diastolic blood pressure, $F(7,294)=8.26, p<$ .0001 ; anxiety, $F(7,294)=4.94, p<.0001$; nervousness, $F(7$, $294)=15.26, p<.0001$; and insecurity, $F(7,294)=8.07, p<$ .0001 . Post hoc $t$ tests in the stress group indicated that compared to baseline, all measures were elevated during the stress phase at +10 and +20 min (all $p s<.001)$ and were back to baseline levels at $+50 \min ($ all $p \mathrm{~s}>.10)$.

An ANOVA with repeated measures for mean cortisol levels showed significant increases over time in the stress group, whereas cortisol levels decreased over time in the control group, $F(7$, $294)=5.76, p<.0001$. Post hoc $t$ tests in the stress group indicated that there was an overall significant difference between cortisol levels right before the TSST $(8.69 \pm 1.05 \mathrm{nmol} / \mathrm{L})$ and the peak cortisol response after stress (13.08 $\pm 1.89 \mathrm{nmol} / \mathrm{L})$ of $50.5 \%, t(21)=3.30, p<.005$, whereas in the control condition there was a significant decrease between cortisol levels right before reading $(8.63 \pm 0.81 \mathrm{nmol} / \mathrm{L})$ and at the end of the reading phase $(7.33 \pm 0.62 \mathrm{nmol} / \mathrm{L})$ of $15.0 \%, t(22)=4.53, p<.0001$. When sex was entered in the repeated measures, a trend-level interaction was found between cortisol, sex, and condition, $F(7$, $287)=1.96, p<.06$. In a post hoc analysis, it appeared that whereas men showed an overall increase of $66.0 \%$ between cortisol levels right before the TSST $(11.06 \pm 1.51 \mathrm{nmol} / \mathrm{L})$ and after the TSST $(18.36 \pm 2.59 \mathrm{nmol} / \mathrm{L}), t(11)=3.48, p<.005$, and women showed only a slight increase of $15.4 \%$ between cortisol levels right before the TSST $(5.85 \pm 0.82 \mathrm{nmol} / \mathrm{L})$ and after the $\operatorname{TSST}(6.75 \pm 0.53 \mathrm{nmol} / \mathrm{L}), t(9)=2.20, p<.06$, no differences

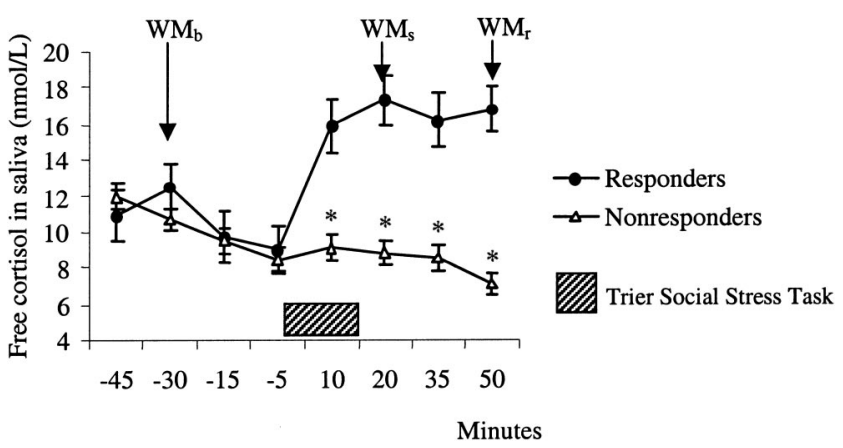

Figure 1. Mean ( $\pm S E M$ ) free salivary cortisol (in nanomoles per liter) before, during, and after the Trier Social Stress Task in responders and nonresponders. $\mathrm{WM}_{\mathrm{b}}=$ working memory at baseline; $\mathrm{WM}_{\mathrm{s}}=$ working memory during stress; $\mathrm{WM}_{\mathrm{r}}=$ working memory during recovery. * significant difference at $p<.05$. were found on any of the cortisol measures between men and women in the control condition.

Because of the high variance of cortisol levels in the TSST group, a post hoc median-split was conducted on the basis of the absolute difference between peak cortisol levels after $20 \mathrm{~min}$ and baseline cortisol levels right before the TSST, at -5 min (median split at $1.90 \mathrm{nmol} / \mathrm{L})$, resulting in a group of cortisol responders $(9$ men and 2 women) and nonresponders ( 3 men and 8 women). Responders showed elevated cortisol levels from right after the TSST until the end of the experiment (from +10 to $+50 \mathrm{~min}$ ) compared with nonresponders, whereas no differences were found between the two groups prior to the TSST (from -45 to $-5 \mathrm{~min}$ ): interaction between group and time, $F(7,140)=4.06, p<.0001$ (see Figure 1). In repeated measures ANOVA analyses with heart rate, blood pressure, and subjective measures as dependent variables, responders did not differ from nonresponders with respect to heart rate, $F(7,126)=0.55, n s$; systolic blood pressure, $F(7$, $140)=0.62, n s$; diastolic blood pressure, $F(7,140)=1.04, n s$, or subjective measures of anxiety, $F(7,140)=0.39$, ns; nervousness, $F(7,140)=2.00, n s$ (trend for responders to be less nervous); or feelings of insecurity, $F(7,140)=0.16$, ns.

\section{Working Memory}

Regardless of condition, working memory performance improved with repeated administration: main effect of time, $F(2$, $84)=4.91, p<.01$. Overall, the stress group did not differ from controls on working memory performance: no main effect of group, $F(1,42)=0.88$, ns. With sex entered as an additional factor, there was no main effect of sex on working memory performance, $F(1,40)=2.20, n s$, regardless of condition. Similarly, within the stress condition, there was no main effect of sex, $F(1,20)=0.01, n s$, or interaction between sex and the three working memory assessments, $F(2,40)=0.42, n s$.

In order to test our main hypothesis on the effects of cortisoladrenergic increases on working memory, we performed a repeated measures ANOVA with working memory as a withinsubjects factor and responders versus nonresponders as a betweensubjects factor. Consistent with our hypothesis, working memory performance of cortisol responders specifically deteriorated during the TSST compared with that of nonresponders, whereas working memory performance of responders did not differ from that of nonresponders at baseline or during the recovery phase: quadratic interaction between group and time, $F(1,20)=4.51, p<.05$ (see Figure 2). When Digit Span forward and backward were analyzed separately, the deterioration of responders during the TSST was significant in the forward condition, $F(1,20)=5.17, p<.05$, but not in the backward condition, $F(1,20)=0.54$, $n s$.

When analyzed separately, participants in the control condition tended to show an overall improvement in working memory performance with repeated administration, similar to the nonresponders in the stress condition, $F(2,42)=2.97, p=.06(M \pm$ $S D$ : first assessment, $47.86 \pm 4.41$; second assessment, $49.55 \pm$ 4.07; third assessment, $53.59 \pm 4.68$ ).

Finally, to analyze which factor was the best predictor of working memory during the TSST, we performed a regression analysis with working memory performance during the TSST as the dependent variable, and sex, increase in cortisol, heart rate, blood pressure, and nervousness as independent variables. With this 


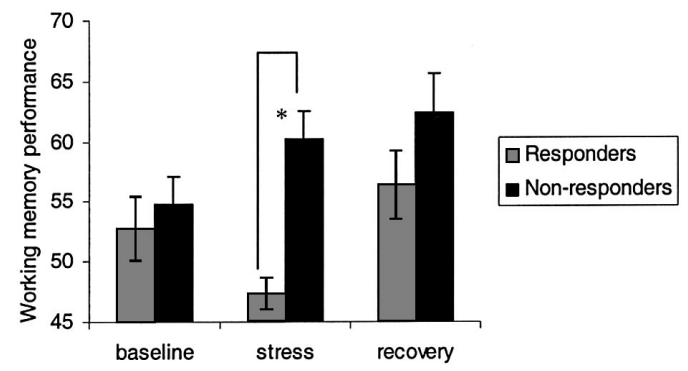

Figure 2. Mean $( \pm S D)$ on working memory performance at baseline, and during the stress and recovery periods of the Trier Social Stress Task for cortisol responders and nonresponders. * significant difference at $p<$ .05 .

model, $43 \%$ of the variance of working memory performance was explained $(r=.66)$. Consistent with the findings of the ANOVA analysis, the best and only significant predictor of working memory performance was the increase in cortisol levels $(\beta=-0.54)$, $t(\mathrm{xx})=-2.23, p<.05$. Sex did not significantly contribute to the working memory performance $(\beta=-0.28), t(19)=-1.17$, ns.

\section{Discussion}

The purpose of the present study was to assess whether the effects of stress-induced cortisol elevations on working memory depend on the level of adrenergic activity during memory performance. Consistent with our hypothesis, we found that working memory was significantly impaired among cortisol responders when they had to perform the memory task in a stressful context (i.e., in front of an audience), during which both cortisol levels and sympathetic activity were enhanced. In contrast, individuals who did not show any cortisol increase in response to the stress task (nonresponders) did not show working memory impairments when performing the task in front of the same audience. In contrast, their performance even improved. Moreover, during the recovery phase, when cortisol levels were still elevated in responders, but the audience had left and sympathetic activity was back to baseline, working memory was no longer significantly impaired in responders. The fact that responders were exclusively distinguishable from nonresponders on the basis of their cortisol responses, and not on the basis of any other stress response, either sympathetic or subjective, suggests that the working memory impairments during stress were primarily associated with stress-induced cortisol levels. The finding that the level of cortisol increase was the best and only significant predictor for working memory performance during stress in a regression analysis supports this idea. Altogether, these findings further substantiate the hypothesis that stress-induced adrenergic activation plays an essential role in the impairing effects of stress-induced cortisol elevations on working memory.

The present findings are remarkably consistent with studies in animals showing that the effects of GCs on working memory depend on the level of stress-induced arousal (Okuda et al., 2004) and adrenergic activity (Roozendaal et al., 2004). The latter study showed that the noradrenergic system, and more specifically the BLA, interacts with the PFC in regulating the effects of GC hormones on working memory functions, and that lesions or pharmacological inactivation of the BLA block the effects of GCs on prefrontal cognitive functions (Roozendaal et al., 2004). The present findings are also consistent with the neurobiological effects of stress exposure on the PFC, as the PFC is a target for both circulating GCs and norepinephrine (NE), and decreased PFC functioning has been reported in relation to both substances (see Arnsten \& Goldman-Rakic, 1998; Lupien \& Lepage, 2001). Increases in NE after stress exposure or administration of anxiogenic drugs have been shown to impair PFC functioning (including working memory) in rats through NE's actions at postsynaptic $\alpha-1$ adrenergic receptors (Arnsten, 1998; Arnsten \& Goldman-Rakic, 1998), which could be reversed by administration of NE $\alpha-2$ agonists (clonidine and guanfacine (Birnbaum, Podell, \& Arnsten, 2000). In the present study, neither sympathetic activation nor cortisol elevations alone resulted in working memory impairments, as not all individuals showed memory impairments during the stress phase (even though all participants had increased sympathetic activation), and no impairments were found in responders during recovery (when cortisol was still elevated). This suggests that in humans, stress-induced cortisol elevations require sympathetic activation for working memory impairments to occur.

The present findings provide an important extension of earlier pharmacological studies showing that (only) high doses of cortisol administration reduce working memory performance in humans (Lupien et al., 1999; Wolf et al., 2001; Young et al., 1999). Lupien et al. (1999), for example, found that only high doses of cortisol administration $\left(600 \mu \mathrm{g} / \mathrm{kg} / \mathrm{hr}^{-1}\right)$ led to impairments of working memory, whereas moderate doses did not. In these studies, working memory was tested in a nonstressful environment, comparable to our third administration of the working memory task. These findings, taken together with the results from our study, could indicate that when acting alone, cortisol may diminish working memory only at extremely high levels, whereas within the physiological range, cortisol may induce working memory only when secreted under conditions of acute stress.

Besides the physiological effects of the stress induction, working memory impairments during acute stress in cortisol responders may also be related to the fact that the task was performed in a stressful context, in front of an audience of three people. Studies in animals have shown that cognitive impairments during stress depend on an interplay between GC responses and the context in which they are elicited, particularly the relevance of the task in relation to its context, the presence of other distracting factors, et cetera (De Kloet et al., 1999). Being engaged in a working memory task in front of an audience can be seen as a form of dual tasking in which metathoughts related to social evaluation may interfere with the memory performance. In the present context, individuals with high cortisol levels may have had more difficulties in inhibiting task-irrelevant thoughts, thereby preempting processing resources and some of the available capacity of working memory. However, responders did not report more anxiety, nervousness, or feelings of insecurity, as may be expected if cortisol activity is related to more intrusive thoughts about the stressful context. In contrast, they even reported being slightly less nervous than nonresponders.

The working memory impairments of cortisol responders during the acute stress phase were most prominent in the Digit Span forward condition, whereas no significant differences were found between responders and nonresponders in the backward condition. Verbal working memory has been hypothesized to consist of two 
subprocesses, that is, maintenance (storage, rehearsal, and matching) and manipulation (reordering or updating) of information, with the Digit Span forward condition primarily assessing maintenance and the backward condition mainly measuring manipulation. Moreover, the maintenance and manipulation subdivisions have been suggested to correspond to distinct roles of the ventrolateral PFC and the dorsolateral PFC in working memory (Fletcher $\&$ Henson, 2001), although recent imaging studies directly comparing maintenance versus manipulation processes in working memory did not always find different brain activation patterns (see Veltman et al., 2003). Because the sample sizes of the cortisol responder and nonresponder groups were relatively small, the specific effects on the Digit Span forward condition should be interpreted with caution. If the present findings are replicated in larger samples, however, this would imply that the maintenance of information is more sensitive to the effects of stress-induced cortisol-adrenergic activation than the manipulation of information. Future studies using functional imaging techniques will be necessary to assess whether the possible differential effects of cortisol-adrenergic activation on the two subprocesses are associated with specific activation patterns in the ventrolateral versus the dorsolateral PFC.

Previous studies have implied that sex and age play important roles in mediating the effects of cortisol on memory, with older women being more susceptible to the effects of GCs (see Seeman, McEwen, Singer, Albert, \& Rowe, 1997; Wolf, Kudielka, Hellhammer, Hellhammer, \& Kirschbaum, 1999), whereas in young populations, men may be more sensitive to the effects of cortisol than women (Wolf et al., 2001). To minimize sex differences in cortisol responses, we excluded from the present study women who were taking oral contraceptives and tested all women in the late luteal phase (Days 21-25) of their menstrual cycle according to self-report, as there are indications that during this phase, stress-induced cortisol levels do not differ between men and women (see Kirschbaum et al., 1999). Nevertheless, mean stressinduced cortisol increases tended to be somewhat larger in men than in women, and as a consequence the responder group contained more men $(n=9)$ than women $(n=2)$. It should first be noted that the memory impairments in the responder group were not due to a simple sex effect on working memory, as no differences were found on working memory performance between men and women in the nonresponders or the control group. Because of the small sample sizes, we could not directly assess whether the working memory performance of female cortisol responders was differently affected by the cortisol increases than those of male responders. With the responders and nonresponders taken together, however, men did not show larger working memory impairments than women, and sex did not contribute as a factor to working memory performance during stress in the regression analysis. Thus, although no sex effects on working memory were found, men were overrepresented in the responder group, suggesting that memory impairments may occur more often in men because men generally show more pronounced stress-induced cortisol increases than women. Additional studies in larger samples are clearly needed to further address whether men and women differ in their susceptibility to stress-induced cortisol effects on working memory, and whether this is dependent on the course of the menstrual cycle.
To our knowledge, this is the first time that the role of acute stress has been studied in relation to the effects of GCs on memory performance in humans. Although studies with larger samples are necessary to further elucidate all the physiological and cognitive factors involved in these impairments, these findings could shed new light on the effects of stress on memory functioning. One of the interesting questions that arises is whether acute stress mediates only cortisol-induced effects on working memory, or whether it also plays a role in mediating the effects of cortisol on declarative memory tasks that depend on hippocampal functions, as would be consistent with findings from studies in animals (Kim, Lee, Han, \& Packard, 2001; Roozendaal, Griffith, Buranday, de Quervain, \& McGaugh, 2003; Roozendaal \& McGaugh, 1997; Roozendaal, Nguyen, Power, \& McGaugh, 1999). So far, two studies in humans are consistent with this idea, reporting selective enhanced delayed recall of emotionally arousing pictures compared with neutral pictures after prelearning cortisol administration (Buchanan \& Lovallo, 2001), and after postlearning cortisol elevations induced by cold presser stress (Cahill, Gorski, \& Le, 2003). A third study did not replicate these findings, however (Rimmele, Domes, Mathiak, \& Hautzinger, 2003). Another interesting implication that needs further investigation is related to the fact that working memory of responders improved when sympathetic activation was back to baseline, suggesting that working memory impairments can be prevented by blocking sympathetic activation. Studies in animals have shown that systemic injections of the centrally acting $\beta$-adrenoceptor antagonist propranolol prevented corticosterone-induced memory impairment (Roozendaal et al., 2004). Future studies investigating these questions will be very relevant, as they may have important implications for the prevention of blackouts and concentration problems during stressful situations such as a job interview or examination, and may provide helpful new venues for the treatment of psychiatric patients with stress-induced prefrontal impairments such as posttraumatic stress disorder or depression.

\section{References}

Arnsten, A. F. T. (1998). Catecholamine modulation of prefrontal cortical cognitive function. Trends in Cognitive Sciences, 2, 436-447.

Arnsten, A. F. T., \& Goldman-Rakic, P. S. (1998). Noise stress impairs prefrontal cortical cognitive function in monkeys: Evidence for a hyperdopaminergic mechanism. Archives of General Psychiatry, 55, 362-369.

Baddeley, A. (1996). The fractionation of working memory. Proceedings of the National Academy of Sciences, USA, 93, 13468-13472.

Birnbaum, S. G., Podell, D. M., \& Arnsten, A. F. T. (2000). Noradrenergic alpha-2 receptor agonists reverse working memory deficits induced by the anxiogenic drug FG142, in rats. Pharmacology Biochemistry and Behavior, 67, 397-403.

Braver, T. S., Cohen, J. D., Nystrom, L. E., Jonides, J., Smith, E. E., \& Noll, D. C. (1997). A parametric study of prefrontal cortex involvement in human working memory. NeuroImage, 5, 49-62.

Buchanan, T. W., \& Lovallo, W. R. (2001). Enhanced memory for emotional material following stress-level cortisol treatment in humans. Psychoneuroendocrinology, 26, 307-317.

Cahill, L., Gorski, L., \& Le, K. (2003). Enhanced human memory consolidation with post-learning stress: Interaction with the degree of arousal at encoding. Learning \& Memory, 10, 270-274

De Kloet, E. R., Oitzl, M. S., \& Joëls, M. (1999). Stress and cognition: Are corticosteroids good or bad guys? Trends in Neuroscience, 22, 422-426.

De Quervain, D. J. F., Roozendaal, B., Nitsch, R. M., McGaugh, J. L., \& 
Hock, C. (2000). Acute cortisone administration impairs retrieval of long-term declarative memory in humans. Nature Neuroscience, 3, 313314.

Fletcher, P. C., \& Henson, R. N. (2001). Frontal lobes and human memory: Insights from functional neuroimaging. Brain, 124, 849-881.

Fuster, J. M. (1997). The prefrontal cortex: Anatomy, physiology, and neuropsychology of the frontal lobe. Philadelphia: Lippincott-Raven Publishers.

Jansma, J. M., Ramsey, N. F., Coppola, R., \& Kahn, R. S. (2000). Specific versus nonspecific brain activity in a parametric N-back task. NeuroImage, 12, 688-697.

Kim, J. J., Lee, H. J., Han, J. S., \& Packard, M. G. (2001). Amygdala is critical for stress-induced modulation of hippocampal long-term potentiation and learning. Journal of Neuroscience, 21, 5222-5228.

Kirschbaum, C., Kudielka, B. M., Gaab, J., Schommer, N. C., \& Hellhammer, D. H. (1999). Impact of gender, menstrual cycle phase, and oral contraceptives on the activity of the hypothalamic-pituitary-adrenal axis. Psychosomatic Medicine, 61, 154-162.

Kirschbaum, C., Pirke, K. M., \& Hellhammer, D. H. (1993). The Trier Social Stress Test: A tool for investigating psychobiological responses in a laboratory setting. Neuropsychobiology, 28, 76-81.

Kirschbaum, C., Wolf, O. T., May, M., Wippich, W., \& Hellhammer, D. H. (1996). Stress- and treatment-induced elevations of cortisol levels associated with impaired declarative memory in healthy adults. Life Sciences, 58, 1475-1483.

Lupien, S. J., Gillin, C. J., \& Hauger, R. L. (1999). Working memory is more sensitive than declarative memory to the acute effects of corticosteroids: A dose-response study in humans. Behavioral Neuroscience, $113,420-430$.

Lupien, S. J., \& Lepage, M. (2001). Stress, memory, and the hippocampus: Can't live with it, can't live without it. Behavioural Brain Research, 127, 137-158.

Lyons, D. M., Lopez, J. M., Yang, C., \& Schatzberg, A. F. (2000). Stress-level cortisol treatment impairs inhibitory control of behavior in monkeys. Journal of Neuroscience, 20, 7816-7821.

McGaugh, J. L., \& Roozendaal, B. (2002). Role of adrenal stress hormones in forming lasting memories in the brain. Current Opinion in Neurobiology, 12, 205-210.

Newcomer, J. W., Craft, S., Hershey, T., Askins, K., \& Bardgett, M. E. (1994). Glucocorticoid-induced impairment in declarative memory performance in adult humans. Journal of Neuroscience, 14, 2047-2053.

Newcomer, J. W., Selke, G., Melson, A. K., Hershey, T., Craft, S., Richards, K., \& Alderson, A. L. (1999). Decreased memory performance in healthy humans induced by stress-level cortisol treatment. Archives of General Psychiatry, 56, 527-533.

Okuda, S., Roozendaal, B., \& McGaugh, J. L. (2004). Glucocorticoid effects on object recognition memory require training-associated emotional arousal. Proceedings of the National Academy of Sciences, USA, 101, 853-858.

Radley, J. J., Sisti, H. M., Hao, J., Rocher, A. B., McCall, T., Hof, P. R., et al. (2004). Chronic behavioral stress induces apical dendritic reorganizations in pyramidal neurons of the medial prefrontal cortex. Neuroscience, 125, 1-6.

Rimmele, U., Domes, G., Mathiak, K., \& Hautzinger, M. (2003). Cortisol has different effects on human memory for emotional and neutral stimuli. NeuroReport, 14, 2485-2488.

Roelofs, K., Elzinga, B. M., \& Rotteveel, M. (2004). The effects of stress-induced cortisol responses and trait avoidance on approachavoidance behavior. Manuscript submitted for publication.

Roozendaal, B. (2000). Glucocorticoids and the regulation of memory consolidation. Psychoneuroendocrinology, 25, 213-238.

Roozendaal, B., Griffith, Q. K., Buranday, J., de Quervain, D. J. F., \& McGaugh, J. L. (2003). The hippocampus mediates glucocorticoidinduced impairment of spatial memory retrieval: Dependence on the basolateral amygdala. Proceedings of the National Academy of Sciences, USA, 100, 1328-1333.

Roozendaal, B., \& McGaugh, J. L. (1997). Basolateral amygdala lesions block the memory-enhancing effect of glucocorticoid administration in the dorsal hippocampus of rats. European Journal of Neuroscience, 9, $76-83$.

Roozendaal, B., McReynolds, J. R., \& McGaugh, J. L. (2004). The basolateral amygdala interacts with the medial prefrontal cortex in regulating glucocorticoid effects on working memory impairment. Journal of Neuroscience, 24, 1385-1392.

Roozendaal, B., Nguyen, B. T., Power, A. E., \& McGaugh, J. L. (1999). Basolateral amygdala noradrenergic influence enables enhancement of memory consolidation induced by hippocampal glucocorticoid receptor activation. Proceedings of the National Academy of Sciences, USA, 96 , 11642-11647.

Rypma, B., Prabhakaran, V., Desmond, J. E., Glover, G. H., \& Gabrieli, J. D. (1999). Load-dependent roles of frontal brain regions in the maintenance of working memory. NeuroImage, 9, 216-226.

Sanchez, M. M., Young, L. J., Plotsky, P. M., \& Insel, T. R. (2000). Distribution of corticosteroid receptors in the rhesus brain: Relative absence of glucocorticoid receptors in the hippocampal formation. Journal of Neuroscience, 20, 4657-4668.

Seeman, T. E., McEwen, B. S., Singer, B. H., Albert, M. S., \& Rowe, J. W. (1997). Increase in urinary cortisol excretion and memory declines: MacArthur Studies of Successful Aging. Journal of Clinical Endocrinology and Metabolism, 82, 2458-2465.

van Aken, M. O., Romijn, J. A., Miltenburg, J. A., \& Lentjes, E. G. W. M. (2003). Automated measurement of cortisol. Clinical Chemistry, 49, $1408-1409$.

Veltman, D. J., Rombouts, S. A., \& Dolan, R. J. (2003). Maintenance versus manipulation in verbal working memory revisited: An fMRI study. NeuroImage, 18, 247-256.

Wechsler, D. (1987). Wechsler Memory Scale—Revised. New York: Harcourt Brace Jovanovich.

Wellman, C. L. (1993). Dendritic reorganization in pyramidal neurons in medial prefrontal cortex after chronic corticosterone administration. Journal of Neurobiology, 49, 245-253.

Wolf, O. T., Convit, A., McHugh, P. F., Kandil, E., Thorn, E. L., Santi, S. D., et al. (2001). Cortisol differentially affects memory in young and elderly men. Behavioral Neuroscience, 115, 1002-1011.

Wolf, O. T., Kudielka, B. M., Hellhammer, D. H., Hellhammer, J., \& Kirschbaum, C. (1999). Opposing effects of DHEA replacement in elderly subjects on declarative memory and attention after exposure to a laboratory stressor. Psychoneuroendocrinology, 23, 617-629.

Young, A. H., Sahakian, B. J., Robbins, T. W., \& Cowen, P. J. (1999). The effects of chronic administration of hydrocortisone on cognitive function in normal male volunteers. Psychopharmacology, 145, 260-266.

Received August 5, 2004 Revision received September 16, 2004 Accepted September 24, 2004 